\title{
Memory for tastes in an operant delayed discrimination
}

\author{
DORIS A. BITLER and ANTHONY L. RILEY \\ The American University, Washington, D.C.
}

\begin{abstract}
The longevity of taste memory was assessed using a simple delayed discrimination procedure. Memory for taste exhibits long-lived properties in conditioned taste aversions, in which delays of up to $12 \mathrm{~h}$ between taste presentation and toxicosis are commonly reported. Although the discrimination procedure utilized here was designed to approximate the parametric features of taste aversion learning (one trial per day, a long period of exposure to the taste, and a salient reinforcer), obtained delays were under $1 \mathrm{~min}$. The differences in taste memory within different procedures are discussed in the context of a retrospective retrieval process for ingestion-related stimuli.
\end{abstract}

Rats can accurately perform simple and conditional delayed discriminations at delays ranging from 20 to $40 \mathrm{sec}$ (see Cohen, Galgan, \& Fuerst, 1986; Weisman, Bruce, \& Beninger, 1987). In marked contrast to these results (which indicate short-lived memory for stimulus events) are the results from conditioned taste aversion (CTA) research (Revusky \& Garcia, 1970; Rozin \& Kalat, 1971; for a bibliography, see Riley \& Tuck, 1985). In the taste aversion procedure, an animal is presented with a novel taste and subsequently made ill. On later exposure to the taste, the animal typically avoids its consumption (see Garcia, Ervin, \& Koelling, 1966). Robust taste aversions have been conditioned with extended delays between taste presentation and toxicosis (e.g., 9-12 h, see Nachman, 1970; Revusky, 1968; Smith \& Roll, 1967; see Etscorn \& Stephens, 1973, for a report of conditioned aversion with a delay of $24 \mathrm{~h}$ between between taste exposure and subsequent toxicosis). In a review of taste aversion learning, Klosterhalfen and Klosterhalfen (1985) advanced the position that the ability of animals to form associations over long delays within the CTA procedure is a function of the specific conditioned stimulus used in taste aversion research, that is, taste. In particular, they noted that taste may have unique properties, one of these being that memory of it is longer lived than is memory of other stimulus modalities (see also Lett, 1979).

On the basis of the assumption that taste memory is long lived, a delayed discrimination employing tastes as discriminative stimuli might be expected to support discriminations at delays approaching those reported in taste aversion learning. Interestingly, using saccharin and sodium chloride as discriminative stimuli for rats in a simple delayed discrimination procedure, Olin and Riley (1986) obtained above-chance performance at average delays of only $45 \mathrm{sec}$. Far from demonstrating the long-lived

\footnotetext{
The research on which this paper was based was supported by a grant from the Mellon Foundation. Requests for reprints should be sent to D. A. Bitler, Psychopharmacology Laboratory, Department of Psychology, The American University, Washington, DC 20016.
}

nature of taste memory, these results closely approximated the delays obtained in delayed discrimination studies employing audiovisual discriminative stimuli (see Cohen, Escott, \& Ricciardi, 1984; Pontecorvo, 1983; Wallace, Steinert, Scobie, \& Spear, 1980). In comparing the discrimination procedure used by Olin and Riley with that utilized in taste aversion learning, several possible explanations for the differences in obtained delays are apparent. Specifically, in the Olin and Riley report, animals were run for 100 trials in each daily session, and the taste stimuli used as discriminative stimuli were relatively small (i.e., $0.01 \mathrm{cc}$ ). The reinforcer on each trial was a 0.02 cc drop of water. In contrast, in CTA procedures, animals are typically given only a single conditioning trial each day, the amount of taste exposure is large (e.g., $10-15 \mathrm{cc}$ ), and toxicosis is a highly salient stimulus. Any one of these factors may be responsible for the different delays obtained in the delayed discrimination and taste aversion procedures (see Barker, 1976; Domjan \& Levy, 1977; Dragoin, 1971; Nachman \& Ashe, 1973; Roberts \& Grant, 1974, 1976).

In Experiment 1, taste memory was assessed in a simple delayed discrimination procedure, the parameters of which more closely paralleled those found in taste aversion learning (e.g., one trial per day, increased exposure to the tastes, and a highly salient reinforcer). The purpose of Experiment 1 was to ascertain whether long delays could be supported within a modified discrimination procedure, allowing for a determination of whether taste memory is long lived in nature (see Klosterhalfen \& Klosterhalfen, 1985) or whether long-delay learning is unique to the taste aversion design.

\section{EXPERIMENT 1}

\section{Method}

\section{Subjects}

Six female Long-Evans hooded rats, approximately 60 days old at the start of the experiment, served as subjects. They were housed in individual, wire-mesh cages and were maintained under a 12:12-h light:dark cycle. Food was available ad lib in their home cages. 


\section{Apparatus}

All experimental sessions were conducted in a $25 \times 30 \times 18 \mathrm{~cm}$ Plexiglas operant chamber, which was enclosed in a box made of soundattenuating material. One wall of the chamber contained three equally spaced holes, which allowed access to stainless steel drinking tubes. A white light was placed $1 \mathrm{~cm}$ below each opening, and a white houselight was centered $9 \mathrm{~cm}$ above the openings. Solenoid valves controlled the delivery of taste stimuli and water reinforcement to the center and side tubes, respectively. Experimental contingencies were programmed on an Apple Ile microcomputer, which also recorded all responses made during the experimental sessions. This computer interfaced with the operant chamber through a Med Associates interface (Model 1080-01). Responses (tube licks) were registered by three Lafayette drinkometers (Model 58008) whenever a circuit was completed between the chamber floor and any of the three drinking tubes. (For a more detailed description of the apparatus, see Mastropaolo, Dacanay, Luna, Tuck, \& Riley, 1984.)

\section{Procedure}

Shaping. Shaping the operant lick response to the two side drinking tubes consisted of allowing the water-deprived subjects to drink only in the operant chamber. A shaping session began with the lights below the left and right drinking tubes turned on, signaling that both tubes were operative. Initially, all lick responses to the drinking tubes were reinforced (i.e., resulted in delivery of a 0.05 -cc drop of water) until the subject had received at least 25 reinforcers on each tube. On subsequent days, a fixed-ratio (FR) 10 schedule of reinforcement was in effect on both tubes, with a $0.05-\mathrm{cc}$ drop of tap water serving as the reinforcer. To prevent perseveration on either side, a forced-choice contingency was added. That is, if an animal took 10 consecutive reinforcers on either side, the light on that side went out and the drinking tube became inoperative until the subject obtained a reinforcer on the opposite side of the chamber. After a subject earned this forced reinforcer, both tubes were illuminated and became operative again. Each shaping session consisted of 50 reinforcers.

Discrimination training. In Condition 1 of discrimination training, each session began with the center stimulus tube illuminated and the houselight on. After the completion of an FR5 on the center tube, each lick at the tube during a 1-sec taste-exposure period resulted in the delivery of a 0.02 -cc drop of taste solution-either sodium saccharin (Sacc, $0.1 \% \mathrm{w} / \mathrm{v}$, Fisher purified) or sodium chloride $(\mathrm{NaCl}, 0.9 \% \mathrm{w} / \mathrm{v}$, Fisher purified). After the taste-exposure period concluded, completion of another FR5 requirement resulted in the center light being turned off and the start of a 1-sec delay period. At the end of this delay, both side lights were turned on. If the taste on the center tube had been Sacc, a single lick on the right tube caused the right light to stay on as the reinforcer was delivered at the right tube, while the light under the left tube went out. Conversely, if the taste on the center tube had been $\mathrm{NaCl}$, a left choice would be reinforced. Reinforcement consisted of 5-sec access to tap water at the appropriate right or left tube, with each lick during this period resulting in the delivery of a $0.05-\mathrm{cc}$ drop. An incorrect choice on either side resulted in the initiation of a 10-sec time-out period, during which all lights in the chamber were turned off. After either reinforcement or time-out, the next trial began. The session terminated after the completion of $\mathbf{5 0}$ trials.

The remaining five conditions of discrimination training differed from Condition 1 in terms of the length of taste exposure, reinforcement and time-out periods, and number of trials per session (see Table 1). In Conditions $1-3$, a criterion of at least $80 \%$ correct discrimination for each taste on 3 successive days was in effect for advancement to the next condition. In Condition 4 , which had only six trials per session, an overall accuracy rate of $80 \%$ on 3 successive days served as the criterion for advancement. In Condition 5, $100 \%$ correct discrimination on 3 days in a row was necessary for advancement to Condition 6, which was a one-trial-per-day discrimination.

Delay testing. As each subject reached Condition 6 (one trial'day), the delay after exposure to the taste was manipulated to determine the longest delay at which each individual subject could correctly perform the discrimination. Correct discrimination on 3 consecutive days constituted a "hit," that is, the ability to accurately use the discriminative stimuli at that delay interval, and the subject progressed to a longer delay. A "miss," or three consecutive incorrect choices, resulted in return-
Table 1

Specific Component Durations and Number of Trials for Each Nondelay Condition of Experiment 1

\begin{tabular}{ccccc}
\hline Condition & $\begin{array}{c}\text { Taste } \\
\text { Exposure } \\
\text { (in sec) }\end{array}$ & $\begin{array}{c}\text { Length of } \\
\text { Reinforcement } \\
\text { (in sec) }\end{array}$ & $\begin{array}{c}\text { Length of } \\
\text { Time-out } \\
\text { (in sec) }\end{array}$ & $\begin{array}{c}\text { Number } \\
\text { of Trials }\end{array}$ \\
\hline 1 & 1 & 5 & 10 & 50 \\
2 & 3 & 8 & 16 & 35 \\
3 & 7 & 20 & 40 & 12 \\
4 & 20 & 55 & 110 & 6 \\
5 & 40 & 120 & 240 & 3 \\
6 & 60 & 720 & - & 1 \\
\hline
\end{tabular}

ing to the last delay interval at which the subject was able to correctly perform the discrimination. A subject was also considered to have missed at a delay if, after 10 successive trials at that delay, no conclusive (i.e., three trials demonstrating either correct or incorrect discrimination) performance was noted. In such a case, the best possible overall performance was only $70 \%$ correct and was attributed to chance. After three hits at a delay interval and three misses at the next longest delay, each animal's participation in the study was concluded.

The precise sequence of delays through which the subjects progressed was titrated individually for each subject according to a number of rules. A hit at a given delay was, generally, followed by the doubling of the delay interval, up to a delay interval of $20 \mathrm{sec}$, after which subsequent delays increased in increments of $4 \mathrm{sec}$. A miss at a delay resulted in a repetition of the last delay at which the subject had a hit. A hit on a delay following a miss resulted in the next delay being the mean of the last hit and miss, and any subsequent hits resulted in succeeding delays of the mean between that delay and the last miss, until either a success was recorded on a previously missed delay or the experiment ended. Two consecutive misses (occurring when a subject missed at a delay on which it had previously hit) were followed by a delay that was the mean of the last missed delay and the preceding hit delay. The specific order of delay presentations for individual subjects is presented in Table 2.

Animals earned all their daily water requirements during the experimental session. If a subject fell below $85 \%$ of its free-feeding weight, supplemental water was given during the time that the animal was usually run in the experiment, thus avoiding either pre- or postsession watering.

\section{Results}

The subjects required an average of 87.83 days to complete the multiple-trial, training phase of the study (Conditions 1-5). Condition 1, the initial training phase, took an average of 33.8 days to complete. Condition 2 required an average of 9.5 days, and Conditions 3-5 took an average of $17.8,18.0$, and 10.0 days, respectively. Subject D5 died before completion of the study; the data for this animal comprise only 57 days of the one-trial-per-day condition (Condition 6). The remaining subjects required an average of 89.8 days of one-trial-per-day discriminations to successfully reach criteria. Due to the individual titration procedure, the precise order of delay lengths varied for each subject. Subjects D1-D4 and Subject D6 were able to perform correct discriminations at maximum delay intervals of $12,49,11,4$, and $3 \mathrm{sec}$, respectively. Subject D5 seemed to be reaching asymptotic performance at a delay interval of approximately $12 \mathrm{sec}$.

\section{EXPERIMENT 2}

Maximum delays obtained in Experiment 1 were under $1 \mathrm{~min}$ for all subjects. The parameters used in Experi- 
Table 2

Sequence of Intervals for Each Subject in Experiment 1

\begin{tabular}{|c|c|c|c|c|c|}
\hline D1 & D2 & D3 & D4 & D5 & D6 \\
\hline $1^{+}$ & $1^{+}$ & $1^{+}$ & $1^{+}$ & $1^{+}$ & $1^{+}$ \\
\hline $2^{+}$ & $2^{+}$ & $2^{+}$ & $2^{+}$ & $2^{+}$ & $2^{+}$ \\
\hline $4^{+}$ & $4^{+}$ & $4^{+}$ & $4^{-}$ & $4^{+}$ & $4^{-}$ \\
\hline $8^{-}$ & $8^{+}$ & $8^{+}$ & $2^{-}$ & $8^{+}$ & $2^{+}$ \\
\hline $4^{+}$ & $16^{+}$ & $16^{-}$ & $1^{+}$ & $16^{-}$ & $3^{+}$ \\
\hline $6^{+}$ & $32^{-}$ & $8^{+}$ & $2^{-}$ & $8^{+}$ & $4^{-}$ \\
\hline $7^{+}$ & $16^{+}$ & $12^{-}$ & $1^{+}$ & $12^{+}$ & $3^{+}$ \\
\hline $8^{-}$ & $24^{-}$ & $8^{-}$ & $2^{+}$ & $14^{-}$ & $4^{-}$ \\
\hline $7^{+}$ & $16^{+}$ & $6^{-}$ & $4^{+}$ & 12 & $3^{+}$ \\
\hline $8^{+}$ & $20^{-}$ & $4^{-}$ & $8^{-}$ & & $4^{-}$ \\
\hline $12^{+}$ & $16^{+}$ & $2^{+}$ & $4^{+}$ & & \\
\hline $16^{-}$ & $18^{+}$ & $4^{+}$ & $6^{-}$ & & \\
\hline $12^{+}$ & $19^{-}$ & $8^{+}$ & $4^{+}$ & & \\
\hline $14^{-}$ & $18^{+}$ & $12^{-}$ & $5^{-}$ & & \\
\hline $12^{+}$ & $19^{+}$ & $8^{+}$ & $4^{+}$ & & \\
\hline $13^{-}$ & $20^{+}$ & $10^{+}$ & $5^{-}$ & & \\
\hline $12^{+}$ & $24^{+}$ & $11^{+}$ & $4^{+}$ & & \\
\hline $13^{-}$ & $28^{+}$ & $12^{-}$ & $5^{-}$ & & \\
\hline $12^{+}$ & $32^{+}$ & $11^{+}$ & & & \\
\hline $13^{-}$ & $36^{+}$ & $12^{-}$ & & & \\
\hline & $40^{+}$ & $11^{+}$ & & & \\
\hline & $44^{+}$ & & & & \\
\hline & $48^{+}$ & & & & \\
\hline & $52^{-}$ & & & & \\
\hline & $48^{+}$ & & & & \\
\hline & $50^{-}$ & & & & \\
\hline & $48^{+}$ & & & & \\
\hline & $49^{+}$ & & & & \\
\hline & $50^{-}$ & & & & \\
\hline & $49^{+}$ & & & & \\
\hline & $50^{-}$ & & & & \\
\hline & $49^{+}$ & & & & \\
\hline
\end{tabular}

+ Successful discrimination. - Unsuccessful discrimination.

ment 1 were chosen to more closely approximate those in the typical taste aversion procedure and were expected to result in longer delays. Since this was not the case, Experiment 2 was run to determine whether the parameters used in Experiment 1 were, indeed, capable of supporting a long-delay taste aversion.

\section{Method}

Subjects

Six female Long-Evans hooded rats, approximately 60 days old at the start of the experiment, served as subjects. They were housed under the same conditions as the subjects in Experiment 1.

\section{Apparatus}

All exposures to tastes and water were conducted in the home cages. Injections were given in a procedure room adjacent to the animal housing area. Food was available ad lib at all times in the home cages.

\section{Procedure}

Adaptation. The animals were given 15-min access to water each day until they were reliably drinking during the first minute of access. All water was acquired in this way. When the animals were drinking immediately after water became available, a water baseline was determined. This was done by allowing access to water for $1 \mathrm{~min}$ and then measuring the amount consumed. A delay of $1 \mathrm{~h}$ was imposed, then water was again available for $10 \mathrm{~min}$. The amount of water consumed during the 10-min access period was also measured.

Taste aversion conditioning. When the baseline of consumption in the 1-min access period appeared stable (i.e., when mean consumption varied by no more than $1 \mathrm{ml} /$ day for 3 consecutive days), taste aver- sion conditioning began. The animals were exposed to one of two tastes during the 1-min access period. The tastes were identical to those experienced by the subjects in Experiment 1 (i.e., either $0.1 \%$ w/v Sacc or $0.9 \% \mathrm{w} / \mathrm{v} \mathrm{NaCl}$ ). Sacc and $\mathrm{NaCl}$ were presented on alternate days. After the 1-min access period ended, there was a 5-min delay. If the taste was Sacc, the subjects received an intraperitoneal (i.p.) injection of $\mathrm{LiCl}(76.8 \mathrm{mg} / \mathrm{kg}$ body weight, $0.15 \mathrm{M})$ at the end of the delay. If the taste was $\mathrm{NaCl}$, the subjects received an equivolume injection of distilled water. Supplemental water was available for $10 \mathrm{~min}$ after each daily session was concluded. This procedure was continued until consumption of the tastes was stable (i.e., mean consumption for either taste varied by no more than $1 \mathrm{ml} /$ day for 3 consecutive days).

\section{Results}

Initial consumption of $\mathrm{Sacc}$ and $\mathrm{NaCl}$ was not significantly different $[T(10)=0.09, p>.05]$. Over conditioning, all subjects decreased Sacc consumption from a mean of $2.96 \mathrm{ml}$ on Day 2 to a mean of $0.38 \mathrm{ml}$ on Day 16 (i.e., all subjects acquired an aversion to the saccharin solution). At the same time, these subjects demonstrated no aversion to $\mathrm{NaCl}$, consuming a mean of $3.00 \mathrm{ml}$ both on the initial exposure to $\mathrm{NaCl}$ (Day 1) and on its final presentation (Day 15). An analysis of variance performed on the subjects as a group yielded significant effects of conditioning $[F(1,15)=65.79, p<.001]$ and days $[F(7,15)=2.97, p<.01]$ and a conditioning $X$ days interaction $[F(7,15)=4.11, p<.001]$, indicating that consumption of the two tastes diverged over the 16 days of the experiment.

\section{DISCUSSION}

In the present experiments, an attempt was made to determine whether tastes serving a discriminative function were long lived when the assessment was made under conditions more comparable to those used in demonstrations of long-delay taste aversion learning. Although length of exposure to the taste stimuli, number of daily trials, and reinforcer salience were more comparable between the two designs, memory for tastes as discriminative stimuli (Experiment 1 ) was clearly not as long lived as memory for tastes in the conditioned taste aversion procedure (Experiment 2).

Although the present studies appear to qualify the conclusion of Klosterhalfen and Klosterhalfen (1985) regarding the long-lived nature of taste memory, it may be possible both to account for the present data and to assume that taste memory is relatively long lived. In order to do so, however, it is necessary to make a different assumption about the processing of stimulus events than is typically made regarding taste stimuli. Specifically, instead of a taste's being proactively processed from the time of its presentation until it is either degraded or used to form an association (e.g., Revusky, 1977), it is conceivable that taste stimuli are stored, soon after presentation, in a specialized memory buffer (Burešova \& Bureš, 1973, 1974). Events occurring later act as retrieval cues that initiate a retrospective scan for recent, ingestion-related experiences. In a recent review of the long-delay learning literature, Domjan (1985) discussed how such a retrospective memory mechanism might account for the reported differences in learning over long delays in different learning procedures. For example, Domjan proposed that different stimuli (e.g., shock, tastes, toxicosis) are differentially effective as retrieval cues. In the case of aversion learning, toxicosis seems especially effective in retrieving the taste stimuli. Once in working memory, the taste can readily form an association with toxicosis, resulting in the acquisition of the conditioned aversion. The failure of most attempts to obtain long delays in traditional learning procedures (as in Experiment 1) may be due to the fact that the stimuli utilized were ineffective as retrieval cues. Thus, although tastes possess the unique characteristic of being maintained in memory storage for long periods 
of time, such that they can be recalled into working memory by appropriate retrieval cues, stimuli vary in their ability to access the stored memory over long delays. Although the specific mechanism underlying the differences between delays supported in different procedures utilizing tastes is not known, it is clear that assumptions about the long-lived nature of taste are not consistent with a traditional account of a prospective processing of taste memory.

\section{REFERENCES}

BARKER, L. M. (1976). CS duration, amount, and concentration effects in conditioning taste aversions. Learning \& Motivation, 7, 265-273.

BureŠovA, O., \& BureS, J. (1973). Cortical and subcortical components of the conditioned saccharin aversion. Physiology \& Behavior, $11,435-439$.

BuREŚvi, O., \& BureŠ, J. (1974). Functional decortication in the CS-US interval decreases efficiency of taste aversion learning. $B e-$ havioral Biology, 12, 357-364.

Cohen, J. S., Escott, M., \& Ricciardi, P. (1984). The role of reinforcement symmetry and stimulus modality in successive delayed matching to sample in the rat. Canadian Journal of Psychology, 38, 63-79.

Cohen, J. S., Galgan, R., \& Fuerst, D. (1986). Retrospective and prospective short-term memory in delayed response tasks in rats. $A n$ imal Learning \& Behavior, 14, 38-50.

Domuan, M. (1985). Cue-consequence specificity and long-delay learning revisited. In M. Domjan (Ed.), Experimental assessment and clinical application of conditioned food aversions (pp. 54-66). New York: New York Academy of Sciences.

Domun, M., \& Levy, C. J. (1977). Taste aversions conditioned by the aversiveness of insulin and formalin: Role of CS specificity. Journal of Experimental Psychology: Animal Behavior Processes, 3, 119-131.

Dragoin, W. B. (1971). Conditioning and extinction of taste aversions with variations in intensity of the CS and UCS in two strains of rats. Psychonomic Review, 22, 303-304.

ETSCORN, F., \& STEPHENS, R. (1973). Establishment of a conditioned taste aversion with a 24-hour CS-US interval. Physiological Psychology, 1, 251-253.

Garcia, J., Ervin, F. R., \& Koelling, R. A. (1966). Learning with prolonged delay of reinforcement. Psychonomic Science, 5, 121-122.

Klosterhalfen, S., \& Klosterhalfen, W. (1985). Conditioned taste aversion and traditional learning. Psychological Research, 47, 71-94.

LETT, B. T. (1979). Long-delay learning: Implications for learning and memory theory. In N. S. Sutherland (Ed.), Tutorial essays in psychology: A guide to recent advances (Vol 2, pp. 1-38). Hillsdale, NJ: Erlbaum.

Mastropaolo, J. P., Dacanay, R. J., Luna, B., Tuck, D. L., \& RILEY, A. L. (1984). Effects of trimethyltin chloride on differentialreinforcement-of-low-rate responding. Neurobehavioral Toxicology \& Teratology, 6, 193-199.
NACHMAN, M. (1970). Learned taste and temperature aversions due to lithium chloride sickness after temporal delays. Journal of Comparative \& Physiological Psychology, 73, 22-30.

Nachman, M., \& Ashe, J. J. (1973). Learned taste aversion in rats as a function of dosage, concentration, and route of administration of LiCl. Physiology \& Behavior, 10, 73-78.

Olin, D. A., \& RIEY, A. L. (1986). Simple delayed discrimination of tastes in the rat. Animal Learning \& Behavior, 14, 405-410.

PonteCorvo, M. J. (1983). Effects of proactive interference on rats' continuous nonmatching-to-sample performance. Animal Learning \& Behavior, 11, 356-366.

REVUSKY, S. (1968). Aversion to sucrose produced by contingent $\mathrm{X}$-irradiation: Temporal and dosage parameters. Journal of Comparative \& Physiological Psychology, 65, 17-22.

ReVuSKY, S. (1977). The concurrent interference approach to delay learning. In L. Barker, M. Best, \& M. Domjan (Eds.), Learning mechanisms in food selection (pp. 319-366). Waco, TX: Baylor University Press.

REVUSKY, S. H., \& GARCIA, J. (1970). Learned associations over long delays. In G. Bower \& J. Spence (Eds.), Psychology of learning and motivation: Advances in research and theory (Vol 4, pp. 1-84). New York: Academic Press.

RILEY, A. L., \& TUCK, D. L. (1985). Conditioned food aversions: A bibliography. Annals of the New York Academy of Sciences, 443, 381-437.

RoberTs, W. A., \& Grant, D. S. (1974). Short-term memory in the pigeon with presentation time precisely controlled. Learning \& Motivation, 5, 393-408.

RoberTs, W. A., \&RANT, D. S. (1976). Studies of short-term memory in the pigeon using the delayed matching to sample procedure. In D. L. Medin, W. A. Roberts, \& R. T. Davis (Eds.), Processes of memory (pp. 79-112). Hillsdale, NJ: Erlbaum.

Rozin, P., \& KAlat, J. (1971). Specific hungers and poison avoidance as adaptive specializations of learning. Psychological Review, 78, 459-486.

Smith, J. C., \& RolL, D. L. (1967). Trace conditioning with X-rays as an aversive stimulus. Psychonomic Science, 9, 11-12.

Wallace, J., Steinert, P. A., Scobie, S. R., \& Spear, N. E. (1980). Stimulus modality and short-term memory in rats. Animal Learning \& Behavior, 8, 10-16.

Weisman, R. C., Bruce, R., \& Beninger, R. J. (1987). Simple and conditional discriminations in the rat: The effects of scopolamine. Learning \& Motivation, 18, 274-287.

(Manuscript received April 3, 1992.) 